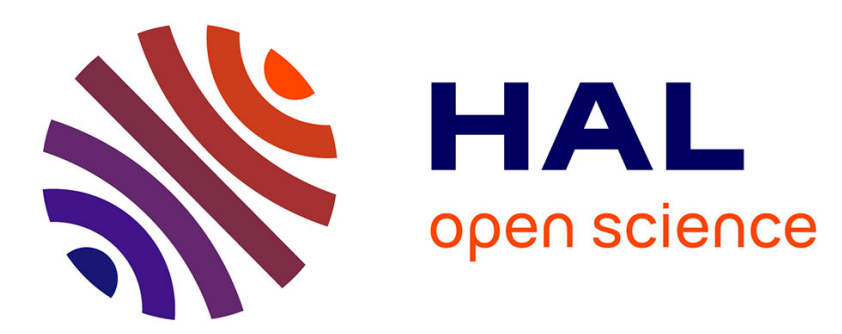

\title{
High temperature packaging for surface acoustic wavetransducers acting as passive wireless sensors
}

Bruno Francois, Jean Friedt, Gilles Martin, Sylvain Ballandras

\section{To cite this version:}

Bruno Francois, Jean Friedt, Gilles Martin, Sylvain Ballandras. High temperature packaging for surface acoustic wavetransducers acting as passive wireless sensors. Sensors and Actuators A: Physical , 2015, 224, pp.6 - 13. hal-02868426

\section{HAL Id: hal-02868426 https://hal.science/hal-02868426}

Submitted on 15 Jun 2020

HAL is a multi-disciplinary open access archive for the deposit and dissemination of scientific research documents, whether they are published or not. The documents may come from teaching and research institutions in France or abroad, or from public or private research centers.
L'archive ouverte pluridisciplinaire HAL, est destinée au dépôt et à la diffusion de documents scientifiques de niveau recherche, publiés ou non, émanant des établissements d'enseignement et de recherche français ou étrangers, des laboratoires publics ou privés. 


\title{
High temperature packaging for surface acoustic wave transducers acting as passive wireless sensors
}

\author{
Bruno FRANÇOIS ${ }^{\mathrm{a}}$, Jean-Michel FRIEDT ${ }^{\mathrm{b}}$, Gilles MARTIN ${ }^{\mathrm{a}}$, Sylvain BALLANDRAS \\ ${ }^{a}$ FEMTO-ST, Time and frequency department, UMR6174 CNRS-Univ. Franche-Comté, Besançon, FRANCE \\ ${ }^{b}$ SENSeOR SAS, c/o FEMTO-ST Time E frequency, Besançon FRANCE \\ ${ }^{c}$ frec-n-sys, Temis innovation, Besançon FRANCE
}

\begin{abstract}
Numerous developments have been dedicated these passed years to demonstrate the use of surface acoustic wave (SAW) devices as passive sensors probed through a wireless radio-frequency link. Giving access to physical parameter variations without embedded power supply, recent works have shown that SAW sensors can be used under harsh environments such as temperatures in excess of $300^{\circ} \mathrm{C}$ and much more. The purpose of this paper is to present a new packaging process for SAW sensors operating under temperature environments up to $600{ }^{\circ} \mathrm{C}$. The robustness of this packaging process is first validated at the above-mentioned temperature using a classical temperature probe via wired connection. The reliability of this process applied to differential SAW sensors then is demonstrated by wireless interrogation of a quartz-based SAW differential sensor from room temperature to $480^{\circ} \mathrm{C}$. The sensor operation has been validated for several tens of hours without major failure nor significant deviation, although the measurement distance dynamic range is observed to be dramatically reduced with operating on such a wide temperature range.
\end{abstract}

Keywords: Packaging process, surface acoustic wave, sensor, high temperature, quartz

\section{Introduction}

Surface Acoustic Wave (SAW) are widely used for telecommunication and on-board signal processing. Their parametric sensitivity also allows one for the development of accurate sensors either based on resonators or delay lines. SAW [1] transducers are therefore considered for probing physical parameters such as temperature in sensing application. Being intrinsically radiofrequency devices compatible with remote powering using RADAR-like measurement strategies, these devices can be used without on-board power supply [2] and as such, the resulting sensors are compatible with harsh environments provided a packaging process adapted to such operation conditions. SAW propagation requires the chip surface to remain stress-free, preventing the use of any kind of material completely surrounding the chip. The most common packaging process for high temperature is based on a mechanical holder for wired measurement $[3,4,5]$. The electrical connections between the chip and the housing are done by wire-bonding using bonding wire material resistant to the targeted operating temperature of the sensor. In [6], a bench dedicated to operating gas sensors in a high temperature environment has been developed, here again with the use of a mechanical holder with high temperature-compatible wires for the electrical connection between the sensor and the measurement setup. Although allowing for an effective characterization of SAW sensors, these approaches are poorly suited to a compact conditioning compatible with actual implementation of the sensor for real environment applications. In the EC/Russia co-funded SAWHOT project (NMP4-SL-2009-247821), SAW sensors have been de- veloped for wireless measurements of temperature up to $700^{\circ} \mathrm{C}$ [7]. In this context, a dedicated packaging process was developed for achieving the targeted sensor capabilities.

One of the principal motivation of the packaging approach developed in the frame of the SAWHOT project was to propose a low cost and simple process to simultaneously achieve the electrical and mechanical connection between the SAW chip and the antenna required for the wireless communication between the reader and the sensor. The considered solution relies on the use of a refractory cement paste covering the whole sensor structure and preserving standard tin soldering from liquefaction and therefore connection defect. The principle of this approach has been first validated using a wired standard Pt100 thermal probe submitted to $600^{\circ} \mathrm{C}$. This packaging process then has been applied to SAW quartz-based sensor for testing its reliability, robustness and reproducibility. As the target of the packaging process was to operate above $600{ }^{\circ} \mathrm{C}$, quartzbased sensors are unsuitable for such high temperatures since the Curie temperature characterizing the onset of the $\alpha$ to $\beta$ crystalline orientation is $573{ }^{\circ} \mathrm{C}[8 ; 9]$. However, some experiments have been done [10] to demonstrate the possibility to use quartz substrates as an alternative solution to pure synthetic substrates i.e. langasite family (LGS) [11, 12] or gallium orthophosphate $\left(\mathrm{GaPO}_{4}\right)$ [13] for temperature from $25^{\circ} \mathrm{C}$ until $480^{\circ} \mathrm{C}$

The paper first briefly recalls the principle of wireless SAW sensors. In order to use SAW sensors built on quartz up to $500{ }^{\circ} \mathrm{C}$, the system needs to be assembled and therefore the packaging process developed in this work is discussed. The final sections are devoted to effective temperature measurements 
in the above-mentioned ranges, assessing the evolution of a sensor chip after temperature cycles, and to the evolution of the physical parameters characterizing the SAW resonator with respect to temperature. The radiofrequency link budget is analyzed in terms of SAW resonator property evolution with temperature: the drop of the quality factor is related to the interrogation range drop, while the coupling coefficient is observed to remain constant. Due to the large resonance frequency shift observed over the extended temperature range, a differential sensor optimizing the use of the radiofrequency spectrum requires overlapping frequency ranges for the two resonances needed for a measurement. The signal processing algorithm used in the wireless reader electronics is flexible enough to allow for the identification of both resonances within an extended frequency band ranging from 430 to $450 \mathrm{MHz}$. A dedicated algorithm is implemented to get rid of the resonance frequency location within preset radiofrequency sub-bands, providing the flexibility to track each acoustic mode despite crossing frequency curves as temperature evolves, and allowing for the measurement of temperature as long as modes do not overlap. As a conclusion, the capability of differential SAW sensors on quartz to measure temperature up to $500{ }^{\circ} \mathrm{C}$ is discussed.

\section{Surface acoustic wave interrogation principle}

SAW sensors are broadly classified either as delay lines [14] or resonators [15], depending on their spectral characteristics, matching at best the requirements of the Industrial, Scientific and Medical (ISM) frequency band they are designed for. Delay lines exhibit a link budget directly related to the electromechanical coupling coefficient and are hence often fabricated on lithium niobate well known for the strength of its piezoelectric properties. The bandwidth required for operating a delay line - typically several tens of megahertz for radiofrequency pulses shorter than $100 \mathrm{~ns}$ - confines their operation in the ISM band centered in $2.45 \mathrm{GHz}$. An alternative approach is the use of narrowband devices (i.e. resonators) which offers access to lower frequency ISM bands. Particularly, the $434 \mathrm{MHz}$ European ISM band is often considered as a tradeoff between antenna dimensions (the quarter electromagnetic wavelength is $17 \mathrm{~cm}$ at such a frequency), dice overall dimensions (typically smaller than $3 \times 3 \mathrm{~mm}^{2}$ ) and cleanroom processing lithography resolution (6 to $9 \mu \mathrm{m}$ acoustic wavelength for most of the available piezoelectric single crystals).

The link budget of a delay line is determined by the electromechanical coupling coefficient $\left(K_{s}^{2}\right)$ of its surface mode wherea the quality factor $Q$ is the most relevant parameter for a resonator. Indeed, in the former case, the RADAR cross-section in the RADAR equation [16], which defines the ratio of the returned power on the incoming power when a target is illuminated, is replaced with the magnitude of the insertion losses $I L$, magnitude of the $S_{11}$ coefficient in the time domain of the echo considered, multiplied by the square of the electromagnetic wavelength $\left(I L \times \lambda^{2}\right)$, representative of typical antenna area intersecting the sphere over which incoming energy spreds. Since the incoming energy is first converted from electromagnetic to acoustic energy through the electromechanical coef- ficient $K_{s}^{2}$ and then from acoustic to electromagnetic energy through the reverse principle involving the same coefficient, $K_{s}^{2}$ is the relevant quantity for a constant acoustic path length, i.e. a constant acoustic propagation loss. On the other hand, in a resonator probing approach, the sensor is first loaded with energy by an incoming monochromatic radiofrequency pulse. The energy storage time constant is $Q / \pi$ periods and therefore conditioned by resonance quality. Once the loading pulse stops, a fixed time delay must be kept before listening to the returned signal to get rid of clutter. Typical durations for unloading passive radiofrequency components and getting rid of clutter are in the microsecond range. The exponential decay with time $t$ of the returned signal is of the shape $\exp (t / \tau)$ with $\tau=Q /(\pi \cdot f), f$ being the resonance frequency of the sensor. Since $20 \cdot \log _{10}(e) \simeq 8.7 \mathrm{~dB}$, the losses associated with exponentially decaying signal returned from the resonator is about $8.7 \cdot f \cdot \pi / Q \mathrm{~dB} / \mu \mathrm{s}$. When working at $f \simeq 434 \mathrm{MHz}$, and considering quality factors of the order of 10000 for quartz resonators, the time constant is $\tau \simeq 7.3 \mu \mathrm{s}$. Recording the signal $1 \mu \mathrm{s}$ after switching from emission to reception yields losses of $1.2 \mathrm{~dB}$ on the RF link balance. The linear dependence of this logarithmic-loss with $Q$ is obvious and will be the core issue discussed in section 6 of the present paper. As mentioned above, quartz-based and langasite-based resonators exhibit $Q$ factors in excess of 10000 at $434 \mathrm{MHz}$ taking advantage of the limited viscoelastic properties of these materials. To conclude this paragraph, quartz-based and langasite-based resonators exhibit $Q$ factors compatible with wireless sensor application because of their low acoustic losses and moderate coupling coefficient (smaller than 1\%), which on the other hand make such materials hardly usable with a delay line approach.

In our embodiment of the pulsed-RADAR like interrogation strategy, the monostatic reader unit emits a radiofrequency signal lasting $5 \tau$ for the spectral width of the emitted pulse to be narrower than the width at half height of the resonator at a variable central frequency which is swept over the ISM band. After each emitted pulse, the returned signal is sampled by a radiofrequency power detector one microsecond after switching from emission to reception, and a total delay of $5 \tau$ including the signal processing duration is waited for before sending the next pulse to make sure that the resonator was fully unloaded and hence make sure that successive probing pulses provide independent samples. The maximum returned signal is associated with the resonance frequency $f$ of the resonator and is related, through a preliminary calibration step, to the physical quantity under investigation. A differential approach in which at least two resonances are probed using this approach is mandatory to get rid of the effects of the transmission channel, local oscillator drift and aging, or sensor aging.

The underlying physical principle of the sensing mechanism is based on the conversion of the incoming electromagnetic energy to acoustic energy using the inverse piezoelectric effect. As piezoelectricity is linear, the finite spectral width of the incoming pulse is defined as a product with the transfer function of the resonator and the returned power is transmitted as an electromagnetic signal through the direct piezoelectric effect. The identification of the resonance frequency 
allows for recovering the physical quantity under investigation via conversion coefficients and physical relations. A parabolic frequency-temperature law is generally used to derive the temperature to which the sensor is submitted [17], whereas linear relations are used for determining torque [18] or pressure [19] via SAW stress sensitivity coefficients. Assuming a properly designed transducer, our investigation focuses on the packaging of the sensor to be operated in a high temperature environment.

\section{Packaging process for high temperature measurements}

For all the experiments, the implemented sensor is the TSEAS10 provided by SENSeOR SAS (Besançon, France), a differential SAW sensor built on quartz using a Copper-doped Aluminum metalization for all electrodes (interdigitated transducers - IDT - and mirrors). The quality factor at room temperature is measured in excess of $Q \geq 8000$ with an electro-mechanical coupling coefficient $K^{2} \simeq 0.02 \%$. Each resonator of the sensor is patterned on the same crystal cut (close to the AT,X cut) but with different propagation directions (Fig.1 (a)) in order to exhibit different frequency behaviors with respect to temperature. The propagation directions have been selected such as each differential frequency corresponds to one and only one temperature, and that each resonance remains in a given frequency band in the temperature range from -20 to $+160{ }^{\circ} \mathrm{C}$. In this range, the average temperature sensitivity of this sensor is about $2500 \mathrm{~Hz} / \mathrm{K}$.

In the current context, we aim at assessing the behavior of such a sensor for measurements at temperatures in excess of $350{ }^{\circ} \mathrm{C}$ (melting temperature of tin-lead solder), meaning that the sensor has to be packaged in order to withstand such temperatures: the main challenge we considered is keeping the antennas connected to the sensor for a wireless measurement. As a possible solution, Gallagher et al.[20] use a meandered dipole antenna printed on a substrate (thermal spray process) with a thick enough layer to allow for the electrical connection with the transducer. Although this solution sounds attractive because no additional connection is needed, it was considered here too expensive and too material consuming for providing an effective solution for industrial applications. Therefore, a more flexible approach has been investigated based on standard packaging approaches modified for withstanding the targeted temperatures.

The sensor is delivered assembled in a $5 \mathrm{~mm} \times 5 \mathrm{~mm} \mathrm{Ky}-$ ocera ceramic package: the sensor chip is glued using a polymer adhesive and wire bonded to the package. While the polymer is unable to withstand temperatures as high as $650{ }^{\circ} \mathrm{C}$, the ceramic package is observed to degrade when exposed between 10 to 20 hours at $650{ }^{\circ} \mathrm{C}$ (Fig.1 (b)). This upper limit is set as the maximum expected survival duration of the sensor exposed to high temperature, and will meet the requirements of testing quartz sensors which will not be used beyond the Curie temperature of $573{ }^{\circ} \mathrm{C}$.

As observed on figure $1 \mathrm{~b}$ ), the metalization layer of the case is oxidized after a couple of hours spent at $650{ }^{\circ} \mathrm{C}$.

The core issue of the packaging is being able to associate the antennas to the ceramic case: our approach is based on a

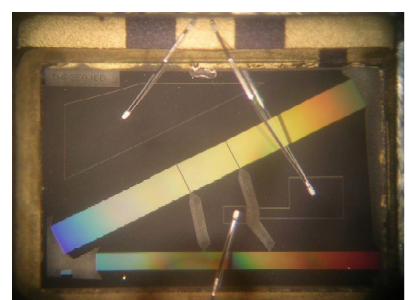

a)

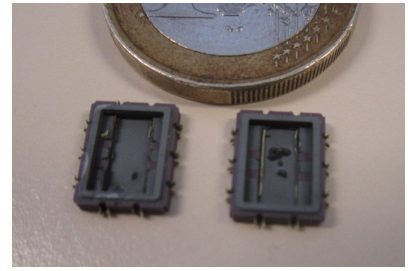

b)
Figure 1: a) Pictures of chip based on two resonators for differential frequency measurements and b) picture of Kyocera case after 20 hours at $650{ }^{\circ} \mathrm{C}$.

tin-lead soldering for providing the electrical contact. Since tin-lead melts around $350{ }^{\circ} \mathrm{C}$, well below the targeted operating temperature, the packaging strategy will aim at confining the liquid tin-lead alloy in order to maintain electrical conductivity even above the melting point. The basic idea is that tin-lead is conductive whatever its physical phase (liquid or solid). Therefore, the problem to solve is keeping the mechanical coherence of the connection on the whole temperature range.

The stainless steel antennas are thus soldered to the ceramic case by using tin-lead solder. A ceramic glue (Ceramabond 503 AREMCO, New York, USA) surrounds the ceramic case containing the sensor and the connection pads in order to provide mechanical strength to the link between the antennas and the ceramic package as well as confine the molten tin-lead solder. Figure 2 shows a sectional drawing of the case containing the quartz chip located in the ceramic package surrounded by the ceramic glue.

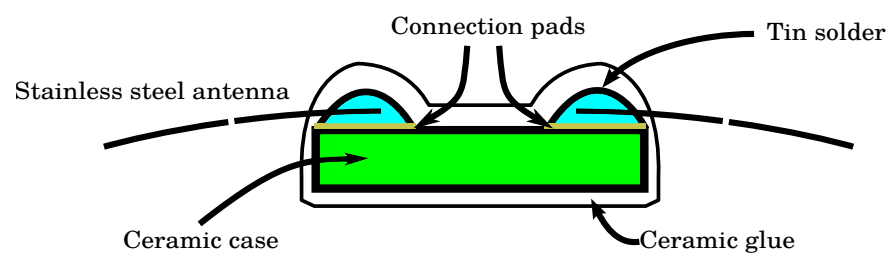

Figure 2: Sectional drawing of the packaged quartz sensor.

The packaging process is tested for mechanical ruggedness and solder confinement with a wired Pt100 temperature probe, packaged by the protocol described previously. Figure 3 shows the probe soldered to the alumina carrier on which two strips of titanium have been preliminary patterned. The probe is then surrounded in the ceramic glue to provide the mechanical strength and prevent electrical connection loss.

The probe has been measured during 3 hours at temperatures in excess of $600{ }^{\circ} \mathrm{C}$ by using the packaging process, demonstrating that the packaging strategy is sound and applicable to SAW sensors.

\section{High temperature measurement setup}

In order to measure SAW sensors, a high temperature test bench has been developed, based on a high temperature ceramic 


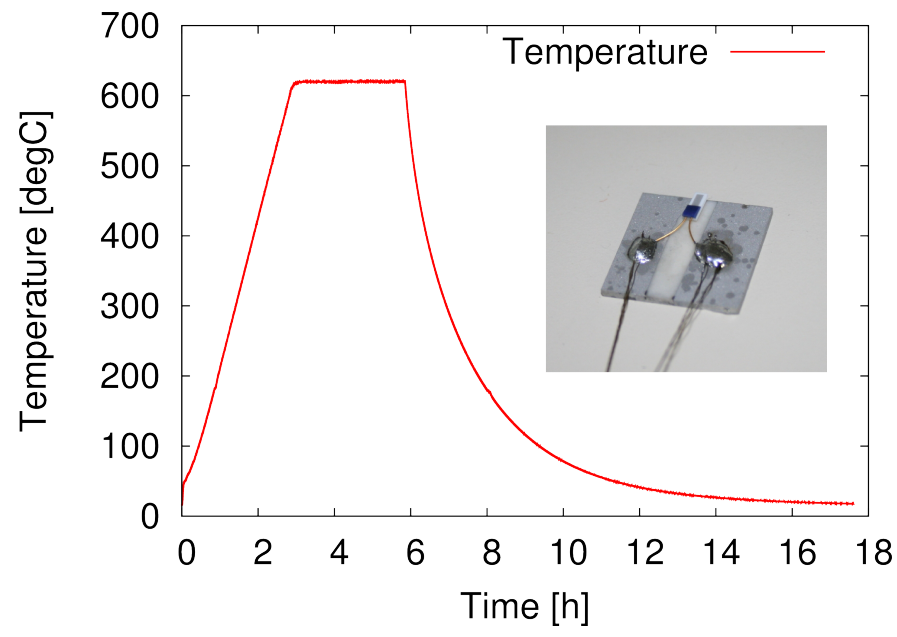

Figure 3: Measurement of the Pt100 resistance during the experiment (3 hours at temperature $>600^{\circ} \mathrm{C}$ ).

oven customized for the application (allowing for sensor interrogation through the back of the oven). A K-type thermocouple acting as temperature reference probe is inserted via a hole at the top of the oven: this temperature sensor is used during the calibration cycles of the SAW sensors. The devices are measured trough one of the panels of the oven, composed of a heat resistant ceramic plate allowing the radiofrequency communication between the reader and the sensor.

\section{Temperature measurement with differential quartz sen- sors}

The TSEAS10 sensor has been designed to comply with a robust measurement strategy in which each resonance is assumed to be confined within a given sub-band of the ISM band. Thanks to this assumption, a one-to-one relation is achieved between the difference of the resonance frequencies and the temperature, and the signal processing software only needs to look for a returned signal within an acceptable power range in each sub-band to identify the resonance frequency. Due to the parabolic dependence of each resonance frequency with temperature, this assumption can only locally be met, and our use of the sensor outside of its designed operational range breaks this assumption: the simulated dependance of each resonance with temperatures up to $500^{\circ} \mathrm{C}$ is shown in Fig.4.

As reported in figure 4 , each resonance frequency is not confined to a preset frequency range, but the frequencies even cross around $200{ }^{\circ} \mathrm{C}$, thus breaking the one-to-one relation between the frequency difference and the temperature and requiring a dedicated measurement algorithm developed below. We note however the existence of a dead zone around the abovementioned crossing temperature for which temperature cannot be determined.

Operating in an extended temperature range implies extending the frequency range probed by the reader, as achieved by using wideband SAW filters rather than filters confined to the $434 \mathrm{MHz}$ ISM band. Beyond the hardware modifications needed

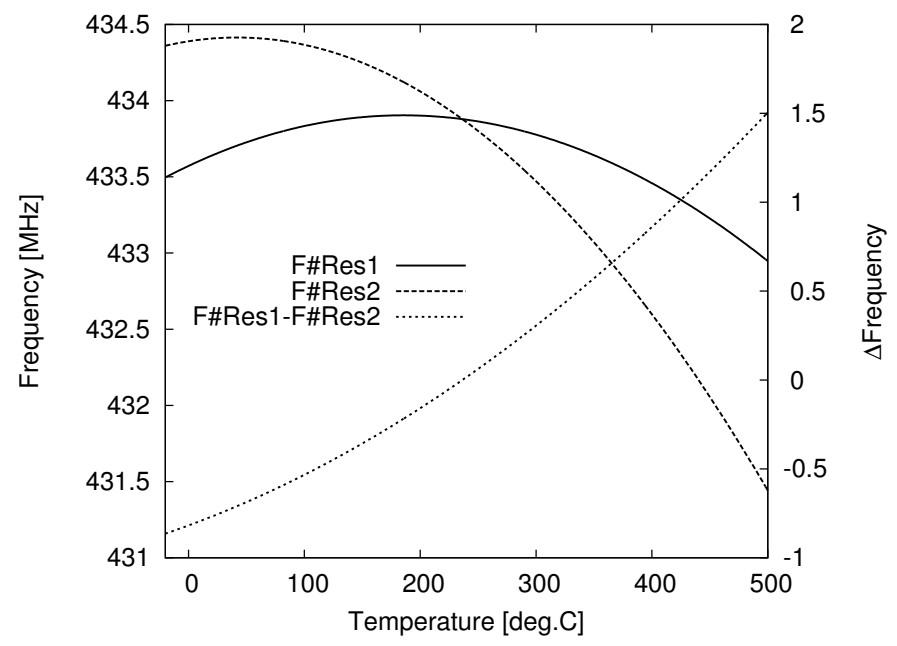

Figure 4: Extrapolation of the behavior of the TSEAS10 frequencies in the temperature range from $-20{ }^{\circ} \mathrm{C}$ to $500{ }^{\circ} \mathrm{C}$.

to operated up to $500^{\circ} \mathrm{C}$, the software can no longer use the assumption of a split ISM band to implement a robust algorithm in which each resonance is assumed to be located in a known subband. Consequently, a flexible algorithm has been developed to probe the whole frequency range from 430.5 to $434.5 \mathrm{MHz}$ and to record the returned power. The frequency sweep step is selected to be equal to one third of the width at half height of the resonance, a tradeoff between immunity to measured power noise and validity of a second order polynomial approximation of the resonance shape [21]. For $Q=6000$, the width at half height $\Delta f$ is $\frac{f}{Q}=\frac{434 \mathrm{MHz}}{6000}=72 \mathrm{kHz}$, and sweeping the $4 \mathrm{MHz}$ wide band with steps of $\Delta f / 3$ requires storing 166 samples to be processed in order to find the two resonance frequencies. We will later show that the quality factor decreases with rising temperature and this sampling step condition is hence always met.

The resonance identification algorithm with no pre-selected sub-band operates as follows: the first resonance is identified as the maximum of the returned power. Considering that the quality factor of each resonance is known, a second measurement is performed excluding the already identified resonance in order to probe the second resonance which is again defined as the maximum of the returned power in this subset of frequencies. This algorithm has proven its robustness for measuring static sensors without the assumption of sub-band division and whatever the quality factor degradation observed as the temperature increases. One drawback is the inability to identify the resonance frequencies when both resonances overlap, i.e. when the frequency difference is closer than the width at half height of each individual resonance. Indeed, with an algorithm based on this principle, the reader is able to measure both resonances when they are separated by at least $3 \Delta f \simeq 200 \mathrm{kHz}$.

As a demonstration of these concepts, a wireless measurement of a SAW sensor up to $450^{\circ} \mathrm{C}$ is performed (Fig.5). For this experiment, the electrical connection between the antennas and the ceramic case containing the SAW chip is achieved by tin/lead solder as in the case of the Pt100 probing test. The 


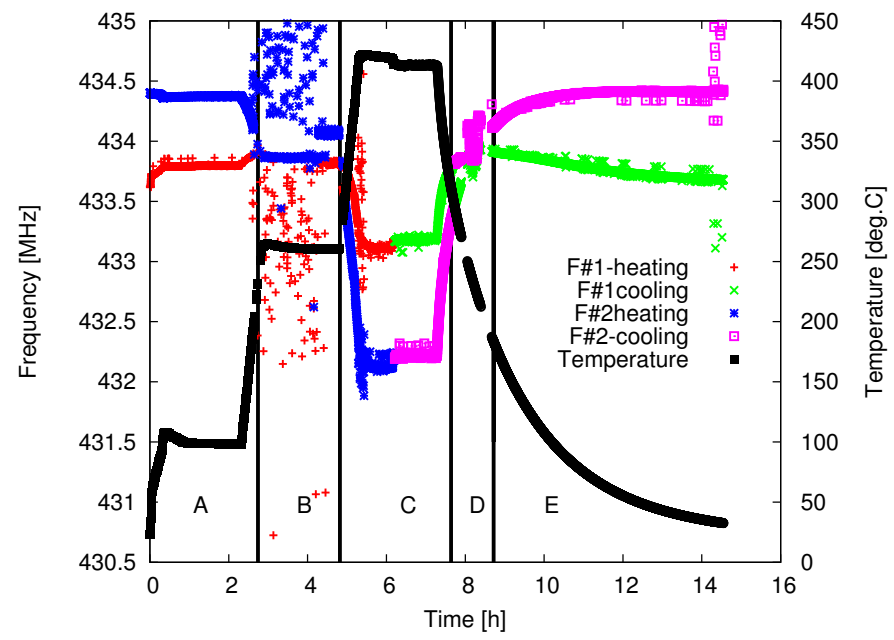

Figure 5: Measurement of the TSEAS10 sensor from room temperature until $450^{\circ} \mathrm{C}$.

whole system is then included in the ceramic paste as described in the section dedicated to the packaging. The experiment can be divided in 5 parts. In parts " $A$ " and "E", the temperature is lower than $200{ }^{\circ} \mathrm{C}$ and they correspond respectively to the heating and cooling phases of the experiment. In parts " $\mathrm{B}$ " and "D", both frequencies are overlapping and the reader is not able to provide any reliable measurement during this period.

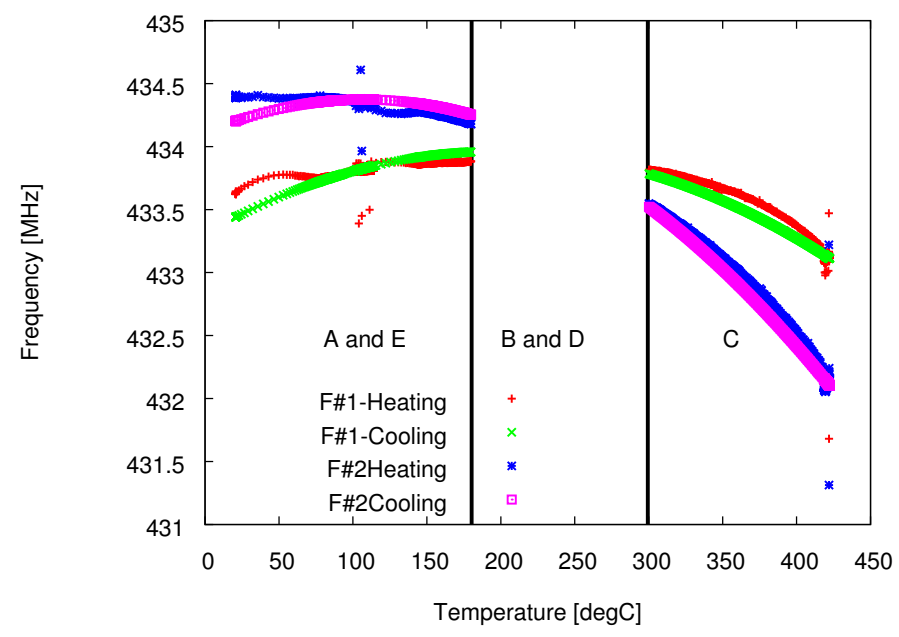

Figure 6: Frequency versus temperature analysis of the recorded data.

Figure 6 emphasizes the inability of the proposed algorithm to probe the resonance frequencies when both values are defined within a $200 \mathrm{kHz}$ band. The intersection of the resonance frequencies reduces the operating range either below or, as is of interest in this article, above the intersection at $200^{\circ} \mathrm{C}$. Despite each individual frequency exhibiting significant $(>100 \mathrm{kHz}) \mathrm{drift}$ during warming and cooling cycles because of transducer aging, either due to degradation and degassing of the organic paste used to glue the quartz chip in the ceramic package, or electrode structure reorganization or oxidation, the differential measurement remains below $90 \mathrm{kHz}$ (in the worst case), yielding a temperature measurement accuracy below $30 \mathrm{~K}$ (considering a sensitivity of $2500 \mathrm{~Hz} / \mathrm{K}$ at ambient) at room temperature, and below $10 \mathrm{~K}$ (considering a sensitivity of $8600 \mathrm{~Hz} / \mathrm{K}$ at $400{ }^{\circ} \mathrm{C}$ ) in the upper temperature range of interest.

\subsection{Visual observation of the chip}

No damage is visually observed on the sensor after exposure for 3 hours at $450{ }^{\circ} \mathrm{C}$ as reported on figure 7 .

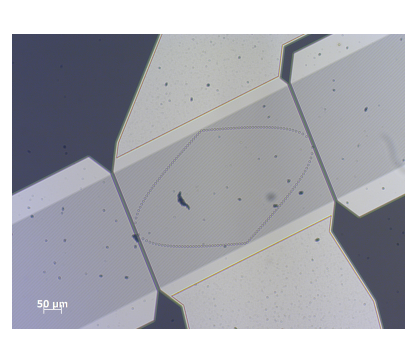

a)

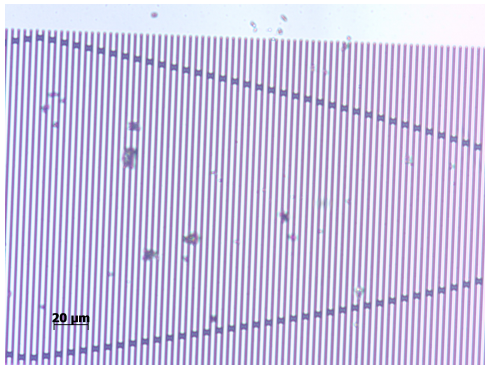

b)
Figure 7: a) General view of one of the resonators and b) closer view of the transducers of the TSEAS10 quartz resonator after 3 hours at $450{ }^{\circ} \mathrm{C}$.

The particles observed on figure 7 come from the milling step needed to open the ceramic package. Despite the melting point of Aluminum $\left(660^{\circ} \mathrm{C}\right)$, the metalization of the IDT seems robust and does not present any visible degradation. Copperdoped $\mathrm{Al}$ metalization is expected to better resist to temperature exposition [22] than pure Al overlays.

\section{Long term aging assessment at $480^{\circ} \mathrm{C}$}

In order to assess the long term stability of the sensor exposed to a temperature above $450^{\circ} \mathrm{C}$, a TSEAS10 sensor packaged in a heat resistance seal as discussed above is placed in the oven at a temperature set to $480{ }^{\circ} \mathrm{C}$. The frequencies of the device are measured as shown in figure 8.

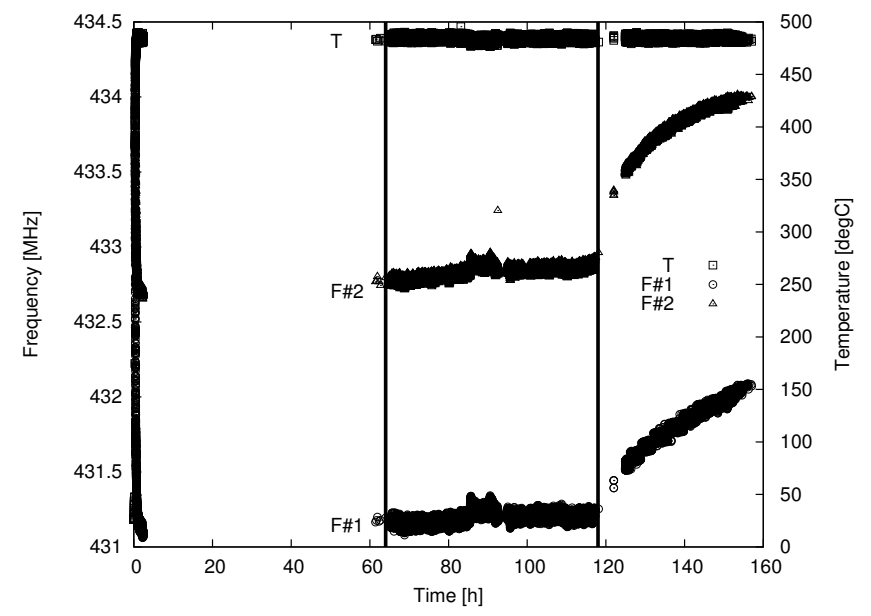

Figure 8: Measurement of both frequencies of the TSEAS10 sensor during 160 hours at $4800^{\circ} \mathrm{C}$. 
At the beginning of the experiment (between 1 and 65 hours), the sensor is not measured due to a loss of returned signal by the reader. A detailed explanation of the reduced interrogation range due to the large quality factor variation is provided later in the data analysis. After adjusting the position of the reader to optimize the radiofrequency link between both elements, the reader is again able to measure the sensor as expected and the device submitted to $480{ }^{\circ} \mathrm{C}$ is still operating after 65 hours. As reported in figure 9 which exhibits a zoom on the measurement results acquired during the first hours of the measurement shown in figure 8 , both frequencies are measured by the reader during the first 1 to 2 hours and the temperature of the oven is stable as observed by a stable signal on both resonance frequencies. Each frequency is associated to the power received by the reader, with Pinc.\#1 and Pinc.\#2 corresponding respectively to F\#1 and F\#2. During the first two hours, the power received from the sensor decreases by $1.5 \mathrm{~dB}$, leading to the loss of both frequency signals.

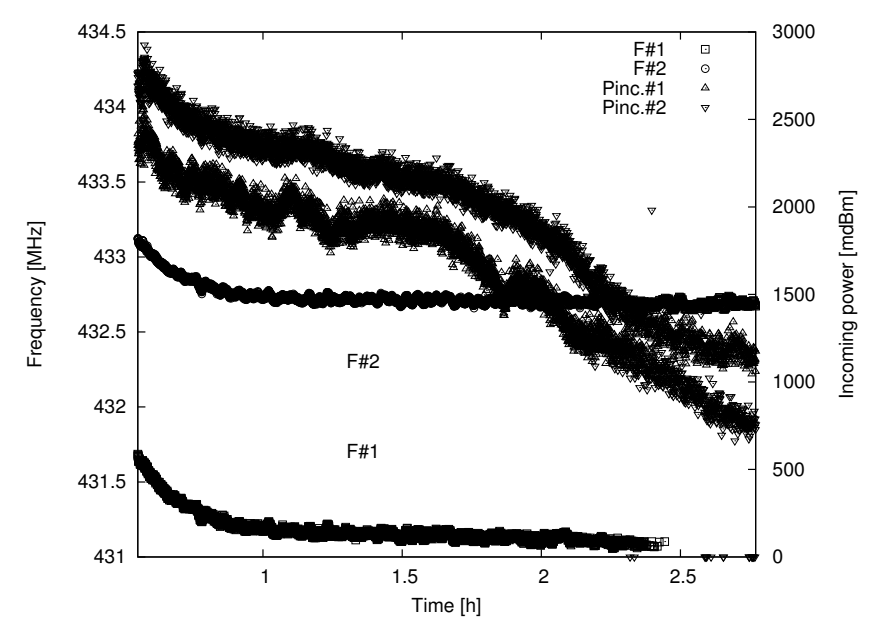

Figure 9: Power received by the reader at the beginning of the experiment reported in figure 8 . Both frequencies are visible and the temperature is stable until the reader is no longer able to gather one of the resonance frequencies.

The fact that the power received from the sensor decreases is representative of the evolution of the physical parameters of the transducers. A resonator is characterized by two quantities beyond the resonance frequency: the quality factor $Q$ and the coupling coefficient $K^{2}$, both involved in the radiofrequency link budget. The evolution of these two quantities, as observed using a measurement using a network analyzer in similar conditions, is reported in Figs.10 and 11. The quality factor and the coupling coefficient are computed every $10 \mathrm{~s}$ and then are associated to the temperature measured by the K-type thermocouple. As shown in figure 10, the quality factor is observed to decrease for each resonator subject to temperatures from $100{ }^{\circ} \mathrm{C}$ to $220{ }^{\circ} \mathrm{C}$. The trend is however not the same for both resonators, although patterned on the same substrate but propagating along different directions: for Res\#1, the quality factor decreases from 6500 to 3200 , while for Res\#2 the drop is from 6300 to 4500 .

Similarly, the coupling coefficient is extracted from the spec-

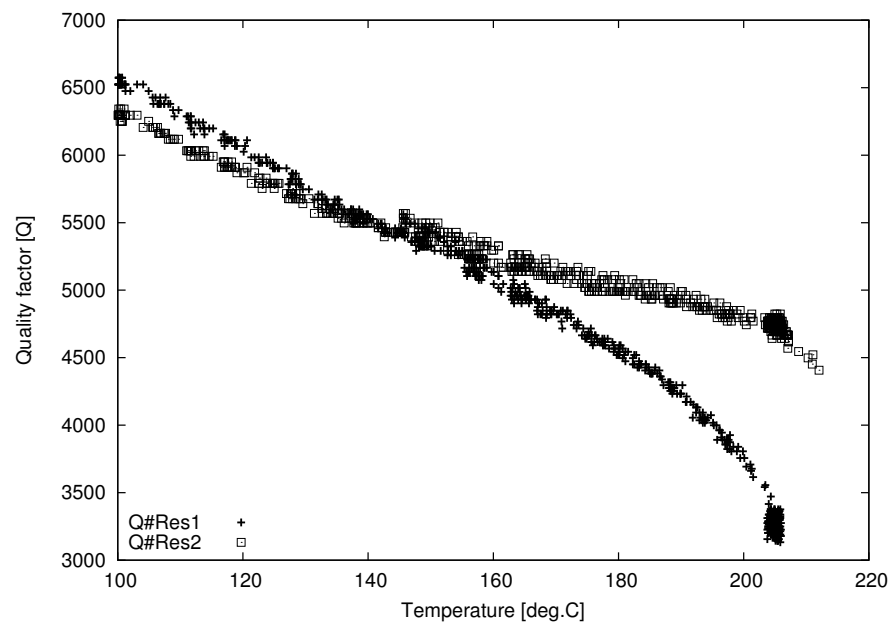

Figure 10: Measure of the quality factor of each resonator versus the temperature between $100{ }^{\circ} \mathrm{C}$ and $220^{\circ} \mathrm{C}$.

tra as shown in Fig.11: this quantity does not evolve significantly over this temperature range, as expected if being associated to an intrinsic property of the piezoelectric substrate.

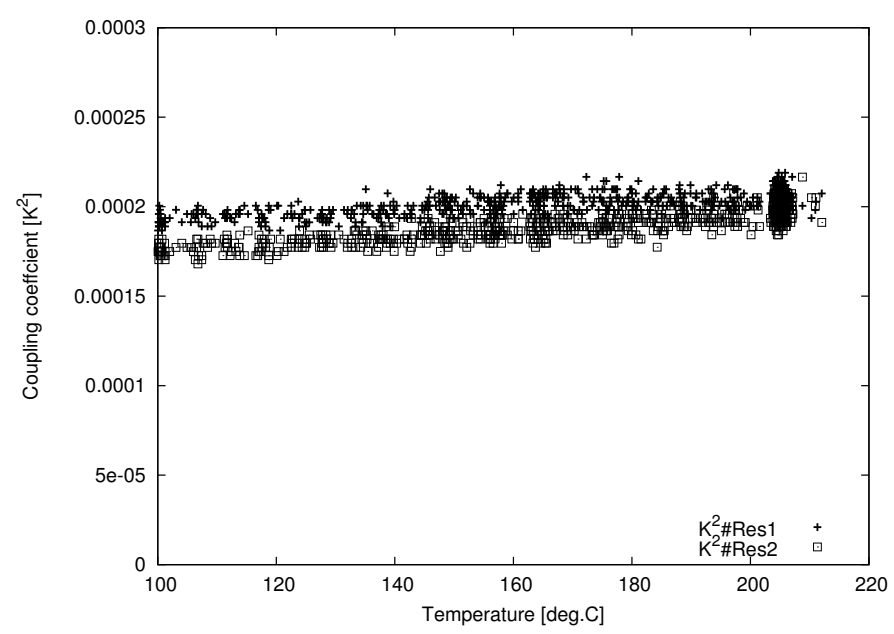

Figure 11: Evolution of the coupling coefficient of each resonator for temperatures ranging from $100{ }^{\circ} \mathrm{C}$ to $220^{\circ} \mathrm{C}$.

Although a decrease in the quality factor induces an increase in the resonance frequency standard deviation [23], we here consider the interrogation range of the reader probing the sensor exhibiting variable $Q$ as being defined by a constant received power on the radiofrequency receiver stage of the interrogation electronics. We focus on the reception stage since the loading stage of the resonator is hardly dependent on the quality factor. During the loading step, an incoming electromagnetic power is received by an antenna. Only a fraction of this incoming energy is converted to acoustic energy due to the impedance mismatch [24]. At resonance, the load resistance on the antenna is dependent on the quality factor through the motional resistance $R$ of the Butterworth-van Dyke model of the acoustic resonator. Since $R$ is related to the coupling coef- 
ficient $K^{2}$, the quality factor, the static capacitance $C_{0}$ and the resonator frequency $f_{s}$ through $[25,26] R=\left(C_{0} \cdot K^{2} \cdot Q \cdot 2 \pi f_{s}\right)^{-1}$, the only significantly varying variable is $Q$. Assuming an antenna impedance $Z_{0} \simeq 50 \Omega$, then varying the quality factor by a factor of 2 yields a reflected power $1-\left(\frac{1-R / Z_{0}}{1+R / Z_{0}}\right)^{2}$ of the order of $2.5 \mathrm{~dB}$. We will see that such a variation is negligible with respect to the decaying exponential signal recorded by the reader unit during the listening step. The exponential decay of the power returned by the resonator, assuming a constant radiofrequency link budget (constant distance and constant antenna efficiency), is affected by the resonator quality factor variation $\Delta Q$ through $\Delta$ loss $=2 \times 8.7 \cdot f \cdot \pi \Delta Q / Q^{2} \mathrm{~dB} / \mu$ s or, since the sampling date after switching from emission to reception is $1 \mu$ s to get rid of clutter, $\Delta$ loss $=2 \times 8.7 \cdot f \cdot \pi \Delta Q / Q^{2} \mathrm{~dB}$. The leading coefficient 2 reflects that our reader electronics receiver stage is based on a power detector [21], a quantity related to the returned voltage by a squared function. The signal loss in the resonator acts in a similar way as the radar cross section in the classical radar equation, and the interrogation range is dependent on this loss through a fourth power law [27]. As an application of the observed quality factor variations during sensor heating from room temperature to $220^{\circ} \mathrm{C}$ (Fig.10), $Q$ varying from 6500 to 3500 yields a degradation of the interrogation range by $17 \%$. Under the same conditions, the coupling coefficient does not significantly vary (Fig.11) and the link budget degradation is solely attributed to the variation of the quality factor. During the cooling phase shown in Fig. 12, with the missing segment as the resonance frequencies cross between 150 and $210^{\circ} \mathrm{C}$, the quality factor rises back from 1950 to 7480 leading to an interrogation range improvement by $67 \%$.

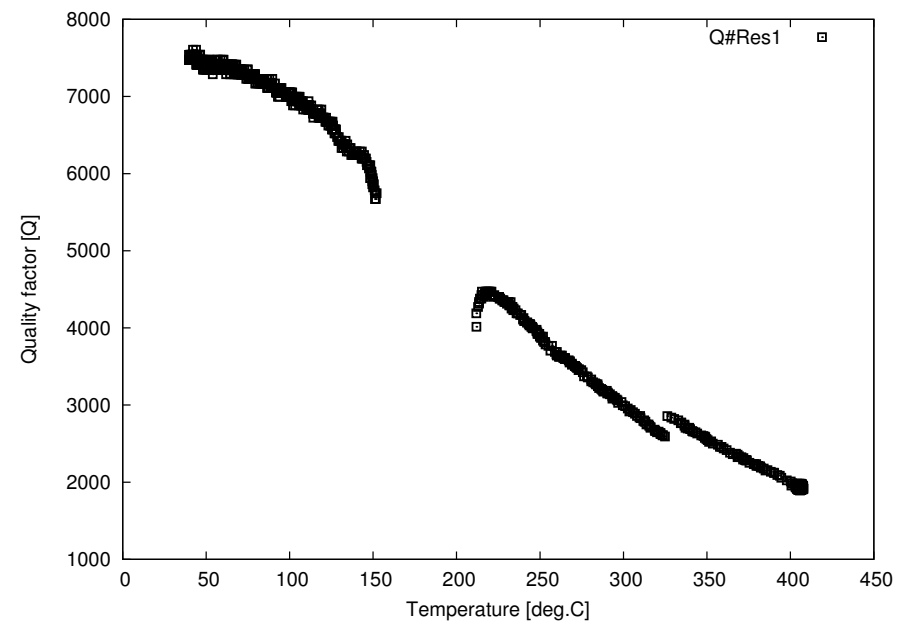

Figure 12: Evolution of the quality factor of one of the resonators as a function of temperature from room temperature to $400{ }^{\circ} \mathrm{C}$.

The analysis of the previous measurements allows for identifying the impact of the temperature on the quality factor of the sensor. As seen in Fig. 9, the quality factor keeps on decaying even at a constant temperature above $450{ }^{\circ} \mathrm{C}$. In order to assess the impact of the quality factor variation on the increase of the power emitted by the reader to compensate the link bud- get degradation, an experiment has been performed under the same conditions but with a sensor wired to a network analyzer and kept at a fixed temperature of $480{ }^{\circ} \mathrm{C}$. The quality factor decrease is assessed for both resonators as reported in Fig. 13. From measurements 1000 to 4000, the quality factor of the first resonator remains almost constant at 1080 after the initial decrease phase observed until point 800 . The quality factor of the second resonator decreases during the whole experiment, leading to a reduction from 1100 to 550 . Such a quality factor variation, with $\Delta Q / Q=0.5$ and $Q \simeq 1100$, yields an equivalent loss of $8.7 \cdot \pi \cdot f \cdot t \cdot \Delta Q / Q^{2} \simeq 5.4$ when sampling at $t=1 \mu \mathrm{s}$ after switching from emission to reception in the wireless reader, consistent with the observed $2 \mathrm{~dB}$ measured in Fig. 9. On the other hand, the associated interrogation range of this sensor has been reduced by $71 \%$, explaining why, once the temperature remains constant, the radiofrequency link between the sensor and the reader is evolving, leading, in the worst cases, to a loss of the radiofrequency signal.

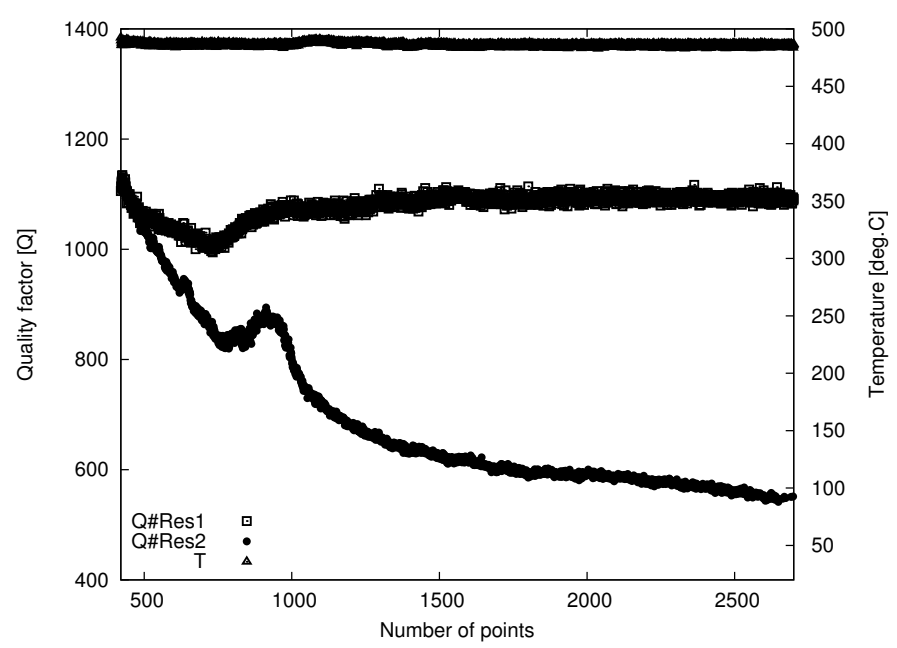

Figure 13: Measurement of the quality factor and the temperature of the sensor while the temperature remains constant at $480{ }^{\circ} \mathrm{C}$.

The frequency measurements reported in Fig. 8 remain stable after optimizing the link budget 60 hours after the beginning of the experiment and keeping the temperature constant at $480^{\circ} \mathrm{C}$. A sudden rise of the frequencies 118 hours after the beginning of the experiment is attributed to a sudden evolution of the electrical parameters of the sensor. Although the reader keeps on recording sensor parameters for another 30 hours before signal loss, the constant drift is analyzed as irreversible aging of the sensing element. A picture of the electrodes of the resonator after completion of this experiment is shown in Fig. 14.

No significant damage to the electrodes is identified visually (Fig. 14), as the particles visible on the surface result from opening the ceramic package, although uniform color change is indicative of surface chemistry modifications of the electrode material and might be the cause of the frequency drift at the end of the experiment reported in Fig. 8.

Hence, the SEAS10 quartz sensor was observed to oper- 


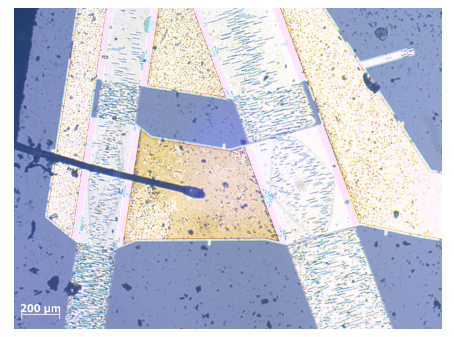

a)

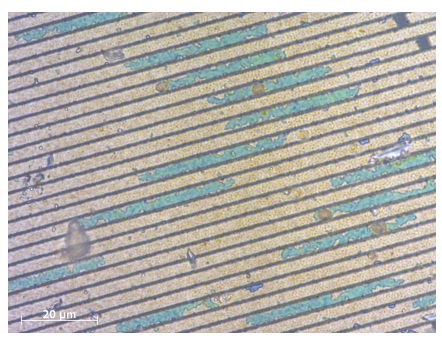

b)
Figure 14: a) Global view of the differential sensor and b) closer view of the transducers of the SEAS10 quartz sensors after 160 hours at $480{ }^{\circ} \mathrm{C}$.

ate at $480{ }^{\circ} \mathrm{C}$ during 118 hours without any major degradation. However, although the temperature remains constant, resonance frequencies drift as reported in figure 8. F\#1 shows a frequency variation of $106 \mathrm{kHz}$, and $\mathrm{F \# 2} 42 \mathrm{kHz}$, leading to a frequency difference variation of $\Delta f=64 \mathrm{kHz}$. Since the temperature sensitivity of the sensor is $8600 \mathrm{~Hz} / \mathrm{K}$ in the temperature range from 450 to $480{ }^{\circ} \mathrm{C}$, the measurement error is estimated to be about $7^{\circ} \mathrm{C}$. The interrogation range is however dramatically reduced to less than $50 \mathrm{~cm}$, from an initial typical range of about $3 \mathrm{~m}$ at room temperature using a monopole antenna on the reader and a dipole antenna attached to the sensor, due to the significant decrease of the quality factor of one of the resonators.

\section{Conclusion}

A packaging strategy compatible with operating temperatures up to $500{ }^{\circ} \mathrm{C}$ has been proposed and applied to surface acoustic wave transducers acting as passive sensors interrogated through a wireless link. In this context, the evolution of the link budget, and most significantly its dependence with the SAW resonator quality factor drop with increasing temperature, is experimentally assessed and analyzed. The electrical connection between the sensing element and the antenna radiators re- 10 mains an issue since the targeted operating temperature range is above the melting temperature of tin-lead solder, which in this approach is confined by the ceramic package even if the conducting link becomes liquid. The reader electronic unit $32 \mathrm{~dB}$ dynamic range on the emitted power allows for a measurement distance of the order of $50 \mathrm{~cm}$ at $480{ }^{\circ} \mathrm{C}$ while compensating for the link budget variation by tuning the emitted power in order to keep the received power at the optimum setpoint of the 13 power detector of -55 to $-65 \mathrm{dBm}$.

Based on these considerations, a quartz-based packaged sensor has been measured from room temperature until $480{ }^{\circ} \mathrm{C}$ and the electrical response has been measured during 118 hours despite the evolution of the physical parameters of the resonator. The quality factor dramatically decreases between room temperature and $400{ }^{\circ} \mathrm{C}$ from 7450 to 1950 , leading to a decrease 16 of the interrogation range of the device by $40 \%$. After a slow drift of the response, the sensor response is irreversibly lost after 160 hours of exposure at $480{ }^{\circ} \mathrm{C}$. As a main result of this study, one can establish that quartz-based differential SAW sensors can be operated above $400{ }^{\circ} \mathrm{C}$ with a low cost packaging solution, even if one should surely not overcome this temperature for long term applications (i.e. more than several hundred hours).

\section{Acknowledgment}

This work was performed within the SAWHOT project, funded in the framework of the European Community's Seventh Framework Program ([FP7/2007-2013]) under grant agreement $\mathrm{n}^{o}$ [NMP4-SL-2009-247821].

\section{References}

1. White, R., Voltmer, F.. Direct piezoelectric coupling to surface elastic waves. Applied Physics Letters 1965;7(12):314-316.

2. Bao, X., Burkhard, W., Varadan, V., Varadan, V.. SAW temperature sensor and remote reading system. In: IEEE 1987 Ultrasonics Symposium. IEEE; 1987:583-586.

3. Fachberger, R., Bruckner, G., Hauser, R., Biniasch, J., Reindl, L., Ruppel, C.. Properties of radio frequency Rayleigh waves on langasite at elevated temperatures. In: Ultrasonics Symposium, 2004 IEEE; vol. 2. IEEE; 2004:1223-1226.

4. Krempl, P., Reiter, C., Wallnofer, W., Neubig, J.. Temperature sensors based on $\mathrm{GaPO}_{4}$. In: Ultrasonics Symposium, 2002. Proceedings. 2002 IEEE; vol. 1. IEEE; 2002:949-952.

5. Bardong, J., Bruckner, G., Franz, G., Fachberger, R., Erlacher, A.. Characterisation setup of SAW devices at high temperatures and ultra high frequencies. In: Frequency Control Symposium, 2009 Joint with the 22nd European Frequency and Time forum. IEEE International. IEEE; 2009:28-32.

6. Thiele, J., da Cunha, M.. High temperature surface acoustic wave devices: fabrication and characterisation. Electronics Letters 2003;39(10):818-819.

7. Franois, B., Sakharov, S., Droit, C., Davis, Z., Richter, D., Fritze, H., Martin, G., Friedt, J., Plessky, V., Brückner, G., Mayer, E., Reindl, L., Karachalios, T., Schiffers, W., Roux, C., Ballandras, S.. Wireless temperature measurements above $500^{\circ} \mathrm{c}$ using surface acoustic wave sensors. In: Procedia Engineering - 26th European Conference on Solid-State Transducers, Eurosensor; vol. 47. 2012:1227-1230.

8. Damjanovic, D.. Materials for high temperature piezoelectric transducers. Current Opinion in Solid State and Materials Science 1998;3(5):469-473.

9. Heising, R.A.. Quartz Crystals for Electrical Circuits. New York: D. Van Nostrand; 1946. (Reprinted 1978, Electronics Industries Association, 2001 Eye St. NW, Washington DC 20006.).

0. da Cunha, M., Lad, R., Moonlight, T., Bernhardt, G., Frankel, D.. High temperature stability of langasite surface acoustic wave devices. In: Ultrasonics Symposium, 2008. IUS 2008. IEEE. IEEE; 2008:205-208.

1. Kaminski, A.A., Silvestrova, I.M., Sarkisov, S.E.,, , Denisenko, G.A.. Investigation of trigonal (La1-xNdx)Ga5SiO14 crystals; ii. spectral laser and electromechanical properties. Phys Stat Sol (A) 1983;80(2):607-620.

2. Hornsteiner, J., Born, E., Fischerauer, G., Riha, E.. Surface acoustic wave sensors for high-temperature applications. In: Frequency Control Symposium, 1998. Proceedings of the 1998 IEEE International. IEEE; 1998:615620.

Krempl, P., Schleinzer, G., et al. Gallium phosphate, $\mathrm{GaPO}_{4}$ : a new piezoelectric crystal material for high-temperature sensorics. Sensors and Actuators A: Physical 1997;61(1):361-363.

4. Bulst, W.E., Fischerauer, G., Reindl, L.. State of the art in wireless sensing with surface acoustic waves. Industrial Electronics, IEEE Transactions on 2001;48(2):265-271.

5. Beckley, J., Kalinin, V., Lee, M., Voliansky, K.. Non-contact torque sensors based on saw resonators. In: Frequency Control Symposium and PDA Exhibition, 2002. IEEE International. IEEE; 2002:202-213.

Skolnik, M.. Radar Handbook, Third Edition. McGraw-Hill Professional; 2008.

7. Friedt, J.M., Rétornaz, T., Alzuaga, S., Baron, T., Martin, G., Laroche, T., Ballandras, S., Griselin, M., Simonnet, J.P.. Surface acoustic wave devices as passive buried sensors. Journal of Applied Physics 2011;109(3):034905. 
18. Kalinin, V., Lohr, R., Leigh, A., Beckley, J., Bown, G.. High-speed high dynamic range resonant SAW torque sensor for kinetic energy recovery system. In: EFTF-2010 24th European Frequency and Time Forum. IEEE; 2010:1-8.

19. Hermelin, D., Daniaux, W., Ballandras, S., Belgacem, B.. Fabrication of surface acoustic wave wireless pressure sensor. In: Frequency Control Symposium, 2009 Joint with the 22nd European Frequency and Time forum. IEEE International. IEEE; 2009:96-99.

20. Gallagher, M., Smith, W., Malocha, D.. An integrated SAW sensor with direct write antenna. In: European Frequency and Time Forum $\mathcal{E}$ International Frequency Control Symposium (EFTF/IFC), 2013 Joint. IEEE; 2013:450453.

21. Friedt, J.M., Droit, C., Martin, G., Ballandras, S.. A wireless interrogation system exploiting narrowband acoustic resonator for remote physical quantity measurement. Review of scientific instruments 2010;81(1):014701014701 .

22. Wojtczak, L.. The melting point of thin films. physica status solidi $(b)$ 1967;23(2):K163-K166.

23. Kalinin, V.. Passive wireless strain and temperature sensors based on saw devices. In: Proc. IEEE Radio and Wireless Conference. 2004:187-190.

24. Kalinin, V.. Modelling of a wireless saw system for multiple parameter measurement. In: Proc. IEEE Ultrasonics Symposium. 2001:1790-1793.

25. J.Vig, . Quartz crystal resonators and oscillators for frequency control and timing applications - a tutorial. In: IEEE International Frequency Control Symposium Tutorials. New Orleans; 2002:.

26. Publication and proposed revision of ansi/ieee standard 176-1987 "ansi/ieee standard on piezoelectricity". IEEE Transactions on Ultrasonics Ferroelectrics and Frequency Control 1996;43(5):717-.

27. Friedt, J.M., Droit, C., Ballandras, S., Alzuaga, S., Martin, G., Sandoz, P.. Remote vibration measurement: A wireless passive surface acoustic wave resonator fast probing strategy. Review of Scientific Instruments 2012;83(5):055001. 